\title{
Prevalence of Helicobacter Pylori in Dyspeptic Patients and Outcome of Load Regimen on its Eradication
}

\author{
Gomaa AA ${ }^{(1)}$, Hassan EA ${ }^{(2)}$, Youssef HSA $^{(3)}$ \\ (1) Ass.Prof And Head Of Departmentof Tropical Medicine \\ Faculty Of Medicine - Fayoum University \\ (2) Lecturer Of Tropical Medicine Faculty Of Medicine - Fayoum University \\ (3) Department of gastroenterology-fayoum general hospital
}

\begin{abstract}
Dyspepsia is a very common upper gastrointestinal problem and its causes may be functional or organic in nature like NSAIDS or H.pylori infection and GERD . also ,H.pylori prevalence is high in dyspeptic patients and because it was considered carcinogenic organism and cause a wide spectrum of health problems (like peptic ulcers, gastric lymphoma ,anemia and thrombothytopenia etc .) . in this study 100 dyspeptic patients investigated for H.pylori prevalence using UGIE to obtain biopsy for histopathology and urease test and revealed 90\% and $89 \%$ prevalence of H.pylori respectively. also H.pylori stool Ag revealed 87\% prevalence in these 100 patients and only urease positive patients ( 89 patients out of 100 dyspeptic patients) included in the study. In this study group female $50(56.2 \%)$ male $39(43.8 \%)$ and before treatment there laboratory investigations revealed anemia in $13(14.6 \%)$ and thrombothytopenia in7 (7.9\%) elevated liver enzymes in 6( 6.7\%) elevated bilirubin in 2 $(2.2 \%)$ and elevated FPS 3 (3.4\%).these 89 patient evaluated after taking load regimen( omeprazole $40 \mathrm{mg}$ once daily ,nitazoxanide 500 mg twice daily ,levofloxacine $250 \mathrm{mg}$ once daily and doxycycline $100 \mathrm{mg}$ once daily ) but with extended duration (14 days ) and after 4weeks from end of treatment H.pylori stool $\mathrm{Ag}$ test revealed eradication of H.pylori in $79(88.8 \%)$ and failure in $10(11.2 \%)$ and there laboratory investigations after treatment revealed increase in HB concentration and number of anemic patients decreased to became $8(9 \%)$, also increased platelets number in some patients reduced thrombocytopenic patients to became 4 (4.5\%), increase in elevated liver enzymes patients in 16
\end{abstract}

$(18 \%)$ and elevated bilirubin in $9(10.1 \%)$ patients and elevated FPS patiens were the same $3(3.4 \%)$ and as a conclusion load regimen was effective and safe with some few advirse effects in treatment of H.pylori infected patients.

key words : H.pylori, load regimen .

\section{Introduction}

Dyspepsia occurs in approximately $25 \%$ of the population each year. It is responsible for substantial health care costs and considerable time lost from work (Shrestha R, et al., 2015).

Dyspepsia is characterized by epigastric discomfort or pain with upper abdominal heaviness or fullness, belching or regurgitation, bloating, early satiation, heart burn, food intolerance, nausea or vomiting (Ali BM, et al., 2015).

Dyspepsia refers to several symptoms of the upper gastrointestinal tract mainly belonging to three patterns: reflux-like, ulcer-like, and dysmotility-like dyspepsia (Vasiliou C, et al., 2014).

In some patients, no specific cause is identifiable for dyspepsia. This group is labeled as functional or non-ulcer dyspepsia (NUD). The major causes of dyspepsia are peptic ulcer disease, gastroesophageal reflux disease, malignancy, and functional dyspepsia. (Ali BM, et al., 2015).

Helicobacter pylori (H. pylori) is an organism that has been recognized as a major cause of gastritis and is associated with duodenal ulcer disease, gastric ulcer disease,gastriclymphoma, and gastric cancer in human. (Peek MR and Blaser MJ,. 2002 ).

Therefore, it is of the utmost importance eradicate the pathogen to reduce $\mathrm{H}$ pylori-related complications( Malfertheiner P. 2002 ). 
The worldwide increasing resistance of H.pylori towards different antibiotics may affect therapeutic management in different countries( De Francesco V,. 2010 ). This will necessitate trials on new antibiotic combinations. There is growing rates of treatment failure with observed marked decline in the eradication rates of triple therapy over the past few decades ( Gisbert JP,. 2000).

The quadruple regimen comprising of proton pump inhibitor, tetracycline, metronidazole, and bismuth is the most commonly used as alternative therapy when failure occurs( Malfertheiner $\mathbf{P}$, 2002).

But despite being cheap and with a success rate of $70 \%$ (Hojo M,. 2001) It has a lot of limitations as lack of compliance of the patients who refuse taking a lot of pills with repeated doses every day in addition to other adverseeffects. So, researches are performed targeting other substitutes. (Chey WD and Wong BCY. 2007). So, guidelines recommend a salvage triple therapy using levofloxacin $500 \mathrm{mg}$ once daily together with PPI and amoxicillin $1 \mathrm{~g}$ twice daily.(Chey WD and Wong BCY. 2007)

Yet, recently the efficacy of levofloxacin-based second-line therapy seems to be decreasing due to an increasing levofloxacin resistance.

( Zullo A,. 2010).

A study by Mégraud et al 2013 on > 2000 European patients with $\mathrm{H}$. pylori infection showed resistance rates of $14.1 \%$ for levofloxacin, $17.5 \%$ for clarithromycin, and $34.9 \%$ for metronidazole. (Megraud F,. 2013 ).

A recent study has found that efficacy of levofloxacin triple therapy has fallen below $80 \%$ in the second-line treatment of H.pylori in Taiwan. (Liou JM,. 2016). Liang et al 2014 reported that the 7-day levofloxacin-containing triple therapy provides an unacceptable perprotocol report card as the second-line treatment for anti-H pylori eradication in Taiwan and recommended modification by either extending the duration to 10 to 14 days or seeking other regimens. (Liang CM,. 2014)

In the hopes of addressing some of these issues We have evaluated LOAD regimen with an extended duration.

\section{Aim of the study}

To study prevalence of Helicobacter pylori in dyspeptic patients and outcome of load regimen on its eradication.

\section{Patients And Methods Study Population:}

After obtaining the approval of the ethical committee at Fayoum faculty of medicine and written informed consents of patients, this prospective study was carried out on 100 patients with dyspepsia (Rome IV criteria) who underwent upper gastrointestinal endoscopy in El- Fayoum general hospital, with consideration of the following inclusion and exclusion criteria.

\section{Inclusion Criteria:}

1. Age more than 18 years.

2. Of 100 dyspeptic patients (Rome IV

criteria) only urease positive patients for H.pylori (89/100) included.

\section{Exclusion Criteria:}

- Patient suffering from liver cirrhosis.

-Patient suffering from renal impairment.

-Pregnant and lactating women.

-Allergy to any of the drugs of the regimen.

\section{Methodology:}

Patients were subjected to:

- Complete clinical assessment and history taking including:

1. The demographic features :age, sex.

2. Risk factors for dyspepsia and H. pylori infection: e g. habits, NSAIDs intake, previous intake of PPIs,...etc.

All patients included in the study were subjected to answer a simplified questionnaire to confirm the presence or absence of the 3 cardinal symptoms (epigastric pain, nausea and vomiting) If there is a discrepancy whether the symptom resolved or not following the treatment offered, These questions were asked initially at the time of diagnosis and in follow up after treatment.

- $\quad$ Routine laboratory assessment including live $r$ and kidney profiles, CBC, blood sugar.

- $\quad$ Also H. pylori stool Ag test was done to all patients $(\mathrm{N}=100)$ and again stool Ag test 4 weeks after LOAD regimen. (bios, microwell diagnostic systems, ref; 10224 , lot;17-ds-233 )

\section{Imaging:}

Abdominal ultrasonography.

- Upper G.I endoscopy: 
Fuji 450 hd and fuji 450 c25 used

1-Preparation: Was done under conscious sedation using a flexible endoscope on subjects who were instructed to fast overnight.

2-Timing: at baseline assessment.

3-Aim:evaluation of dyspepsia and confirm $H$. pylori diagnosis.

4-The endoscopist documented the following :

- $\quad$ Presence of gastritis.

- Any associated endoscopic finding e.g.: hiatus hernia, peptic ulcer,...etc.

- Biopsies for histopatholgic and urease test diagnosis of $H$. pylori: three random biopsies were obtained from each of the antrum and the body of the stomach and separetly sent to be investigated.

\section{- Histopathology:}

All biopsies were examined by a single expert pathologist who was blinded for clinical and endoscopic data of the patients.

Biopsy specimens were used to assess pathologic changes associated with $H$. pylori infection according to the visual analogue scale of the updated Sydney classification including: degree of chronic inflammation, activity of gastritis, atrophy, density of $H$. pylori colonization, intestinal metaplasia and malignancy .

- $\quad$ All study group patients (89) were received LOAD regimen for 14 days, followed up during and after treatment then reevaluated using stool Ag test.

\section{Results}

The present study included 100 patients with dyspepsia who were assessed by upper gastrointestinal endoscopy, in El- Fayoum general hospital .

\section{Patients characteristics:}

- Age/sex characteristics of the study participants $(\mathrm{N}=100)$ :

Age ranged from 19-58 years . the Mean \pm SD was 36.6 \pm 10.2 .

females were 58 patients $(58 \%)$ and Males were 42 patients $(42 \%)$.

\begin{tabular}{|l||c||c|}
\hline \multicolumn{1}{|c||}{ Variable } & Mean \pm SD & Range \\
\hline \hline$\underline{\text { Age } \text { (years) }}$ Variable & $36.6 \pm 10.2$ & $19-58$ \\
\hline \hline \multicolumn{1}{|c||}{ Sex: } & & $\%$ \\
Female & 58 & \\
Male & 42 & 48.0 \\
\hline
\end{tabular}

\section{Presenting symptoms:}

Presenting complaints were epigastric pain, nausea and vomiting 46 (46\%), only epigastric pain $26(26 \%)$ and nausea and/or vomiting were $28(28 \%)$.

\begin{tabular}{|l|c||c|}
\hline \multicolumn{1}{|c|}{ Variable } & N & \% \\
\hline Complaint: & & \\
Epigastric pain & 26 & 26.0 \\
Nausea \& vomiting & 28 & 28.0 \\
Epigastric pain + nausea \& vomiting & 46 & 46.0 \\
\hline
\end{tabular}

\section{Prevalence of HP :}

According to HP stool Ag test there were 87(87\%) positive and 13(13\%) negative .

According to multiple gastric biopsies histopathology 90 (90\%)

Were positive and $10(10 \%)$ were negative .

According to rapid urease test $89(89 \%)$ were positive and $11(11 \%)$ were negative for H.pylori infection . 


\begin{tabular}{|l||c||c||}
\hline \multicolumn{1}{|c||}{ Variable } & N & \% \\
\hline \hline Antigen: & 87 & 87.0 \\
+ve & 13 & 13.0 \\
-ve & & \\
\hline Biobsy: & 90 & 90.0 \\
+ve & 10 & 10.0 \\
-ve & & \\
\hline \hline Urease: & 89 & 89.0 \\
+ve & 11 & 11.0 \\
\hline -ve
\end{tabular}

\section{Rate of eradication of H.pylori after treatment :}

after LOAD regimen on H.pylori infected patients eradication rate was $\mathbf{7 9}(\mathbf{8 8 . 8 \%})$ eradicated and $10(\mathbf{1 1 . 2 \%})$ non-eradicated

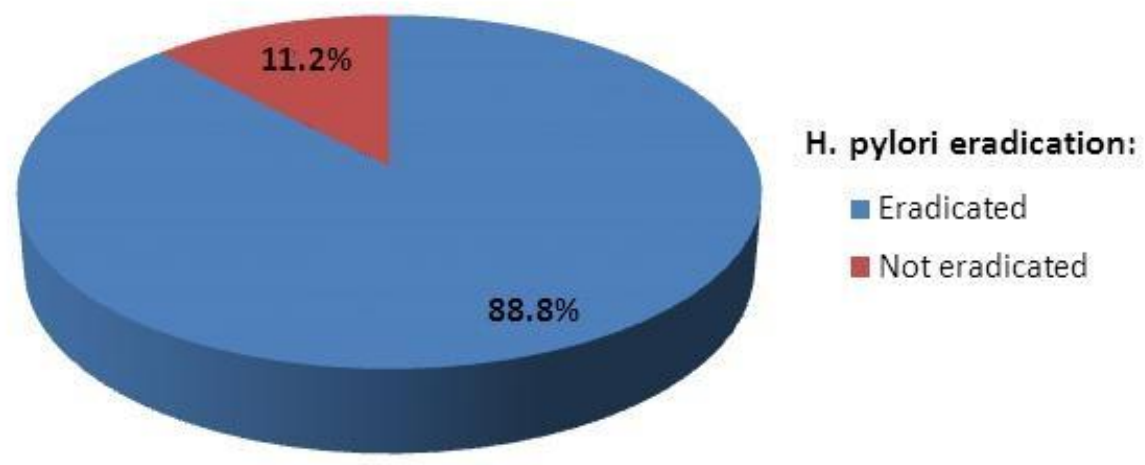

\section{Discussion}

Dyspepsia occurs in approximately $25 \%$ of the population each year. It is responsible for substantial health care costs and considerable time lost from work.

Dyspepsia is characterized by epigastric discomfort or pain and can be associated with upper abdominal heaviness or fullness, belching or regurgitation, bloating, early satiation, heart burn, food intolerance, nausea, or vomiting. The lower bowel function is usually not affected. (Ali BM, et al., 2015).

There are two types of dyspepsia: organic and functional. The main causes of organic dyspepsia are: gastroesophageal reflux, chronic peptic ulcer disease. (Tacikowski T, et al., 2017).
The present study included 100 patients with dyspepsia whom were assessed by upper gastrointestinal endoscopy and biopsy taken for rapid urease test and histopathology also $\mathrm{H}$. pylori stool Ag test was done to find out prevalence of $\mathrm{H}$. pylori in these dyspeptic pateints.

The mean age was $36.6 \pm 10.2$ which correlates with study of Shrestha $\mathbf{R}$ et al. (2015) had reported a younger age which was 39.37 years \pm 18.16 years.

On the other hand, Gado A et al. (2015) showed that the mean age was $43 \pm$ 15 years, Ali BM et al. (2015) showed that the mean age was $44.70 \pm 18.04$ years and Faintuch JJ et al. (2014) showed that the mean age was 44 years. 
The main presenting complaints were epigastric pain, Nausea and/or vomiting $46 \%$ (46 patients ) that result correlated to Faintuch JJ et al. (2014) who showed that presenting symptoms were epigastric pain in $68 \%$ of the patients and $32 \%$ with post-prandial fullness.

In the present study Hilecobacter pylori prevalence was $89 \%$ (accourding to Urease test) of 100 dyspeptic pateints and this is correlated to Khedmat et al., 2013 who showed that prevalence is $84.8 \%$ and disagreed with Alazmi et al, 2010 who showed that prevalence is $65.7 \%$ andThese differences are most likely associated with the testing methods, socio-economic status of the study populations, and geographical variation in the prevalence of infection. (Khedmat H, et al., 2013)

As for gender in the present study, males were 42 patients (42\%) and females were 58 patients $(58 \%)$. These results showed that the incidence of dyspepsia is higher in females than males, which is in agreement with the findings of GarcíaAlonso FJ (2016) who showed that the males were $38.8 \%$ and females were $61.2 \%$, Ali BM et al. (2015) who showed that $46 \%$ were males and 54\% were females, Shrestha $R$ et al. (2015) showed that males were $46.52 \%$ and females $53.48 \%$ and Faintuch JJ et al. (2014) who showed that males were $35 \%$ of the patients and females were $65 \%$.

On the other hand, the results of the present study disagreed with Gado A $\boldsymbol{e t}$ al . (2015) who showed that the incidence of dyspepsia is higher in the males $(51 \%)$ than females (49\%) and Paul Set al. (2015) who showed that males were $56.7 \%$ and females were $43.3 \%$.

There are many explanations that findings of dyspepsia are more common in females, Flier SN, Rose S (2006) showed that in dyspepsia especially functional dyspepsia there are gender-specific features of functional dyspepsia, gender-related differences in gastric emptying was found more delayed in women, also women show more proximal gastric relaxation than males using the gastric barostat, show different psychosocial factors, also women tend to have lower thresholds of pain perception when compared with men and they tend to be less tolerant of the stimuli than men and there may be a peri-menstrual hypersensitivity.

Also, Napthali $\mathrm{K}$, et al. (2016) showed that females are more likely to report symptoms and continue to report symptoms over time. Women also experience greater distress from this condition compared with males and have higher associations with psychosocial factors .

Helicobacter pylori is a human pathogen that causes chronic gastritis, plays a causative role in gastric and duodenal ulcer and is involved in gastric carcinogenesis. It has been classified as a definite (Class I) carcinogen of humans. This gram negative gastric pathogen is also regarded as being a possible important factor in at least a subset of patients with functional dyspepsia. (Tiwari,et al., 2011)

According to,Hunt, et al .2011 Globally, different strains of $\mathrm{H}$. pylori appear to be associated with differences in virulence, and the resulting interplay with host factors and environmental factors leads to subsequent differences in the expression of disease. Age, ethnicity, gender, geography and socioeconomic status are all factors that influence the incidence and prevalence of $\mathrm{Hp}$ infection. (Hunt RH, et al., 2011)

In a systematic review and meta-analysis to assess the prevalence of $H$. pylori infection worldwide, we observed large amounts of variation among regionsmore than half the world's population is infected and prevalence in egypt was more than $40.9 \%$ in general population .( James K., et al., 2017)

As regard gender, in the current study hilecobacter pylori prevalence in female was $50(56.2 \%)$ higher than in males 39(43.8\%) .

In our study, significant difference was observed in gender . female had a higher infection rate than male. The explanation for this difference can only be 
speculative at this moment, being most probably related to the hormonal differences between the two genders, studies identified an important role of oxytocin in the gastric evacuation rate (GER). (J. Borg, et al., 2009)

Accurate diagnosis of Helicobacter pylori (H. pylori ) infection is a crucial part in the effective management of many gastroduodenal diseases. Several invasive and non-invasive diagnostic tests are available for the detection of $\mathrm{H}$. pylori and each test has its usefulness and limitations in different clinical situations. Although none can be considered as a single gold standard in clinical practice, several techniques have been developed to give the more reliable results. Invasive tests are performed via endoscopic biopsy specimens and these tests include histology, culture, rapid urease test as well as molecular methods. Developments of endoscopic equipment also contribute to the real-time diagnosis of $\mathrm{H}$. pylori during endoscopy.

Urea breathing test and stool antigen test are most widely used non-invasive tests, whereas serology is useful in screening and epidemiological studies. (Yao-Kuang Wang, et al., 2015)

Histology is usually considered to be the gold standard in the direct detection of $H$. pylori infection and is also the first method used for the detection of $H$. pylori. However, several factors influence the diagnostic accuracy of histology, such as site, size and number of biopsies, staining methods, proton pump inhibitor (PPI), antibiotics and experience of the examining pathologist. (Yao-Kuang Wang, et al., 2015)

Peptide nucleic acid fluorescent in situ hybridization(PNA-FISH), which can be used on histological preparations, is a highly sensitive (97\% sensitivity) and specific (100\% specificity) technique for the diagnosis of $H$. pylori infection. PNAFISH can identify coccoid form of $H$. pylori which is usually undetectable by routine histological exam because this method could avoid individual biasness from morphological identification .( Yilmaz O, and Demiray E. 2007)

For routine clinical practice, rapid urease test (RUT) is the most useful invasive test for the diagnosis of H.pylori infection because it is inexpensive, rapid, easy to perform, highly specific and widely available . in general, according to Vaira, et al . 2010 the commercial rapid urease tests have sensitivity above $85 \%-95 \%$ and specificity above $95 \%-100 \%$. (Vaira D, et al., 2010)

That was parallel to the current study, urease test had sensitivity above $97.8 \%$ and specificity above $100 \%$.

Stool antigen test (SAT) a noninvasive method with good sensitivity and specificity, 94\% and 97\% respectively in global meta-analysis, in the diagnosis of H. pylori infection .(Gisbert JP, de la Morena $F$ and Abraira V., et al . 2006)

In current study, when comparing H.pylori stool $\mathrm{Ag}$ to histology sensitivity,specificity and total accuracy were $93.3 \% \quad(86.2-96.9 \%), \quad 70 \% \quad$ (39.7$89.2 \%)$ and $91 \%(83.8-95.2 \%)$ respectivly. When compared to urease test sensitivity, specificity and total accuracy were $93.2 \%$ $(86.1-96.9 \%), 63.6 \%(35.4-84.8 \%)$ and $90 \%(82.6-94.5 \%)$.

Also, in the current study we have studied outcome of LOAD regimen (ppi double dosage 40mg once, nitazoxanide $500 \mathrm{mg}$ twice,levofloxacine $250 \mathrm{mg}$ once and doxycycline $100 \mathrm{mg}$ once for 7-10 days ) but with extended duration course (14 days ) on eradication of HP.

Several regimens had been recommended for HP treatment but still with high failure rate more than 20\%. (GisbertJP, and Pajare JM. 2002)

Because of high resistance to clarithromycin and metronidazole and also worldwide lack of availability to bithmus salts so there is a great challenge to current management of first- line eradication failures. (Romano M, et al., 2008)

Also, A study by Mégraud, et al. 2013 on $>2000$ European patients with $\mathrm{H}$ pylori infection showed resistance rates of $14.1 \%$ for levofloxacin, $17.5 \%$ for 
clarithromycin, and $34.9 \%$ for metronidazole. (Megraud F, et al., 2013 ).

However ,efficacy of levofloxacin based second-line therapy seems to be decreasing due to an increasing levofloxacin resistance.( Carothers JJ, et al., 2007)

In our study, successful eradication of HP was $\mathbf{8 8 . 8 \%}$ (79/89 patients) .

Molina-Infante et al 2015 also found that high-dose acid suppression and 14-day duration of treatment can improve eradication rates by up to $10 \%$.(MolinaInfante J, et al., 2015)

These results are parallel to those of the study by Basu et al. 2011on treatment naive $\mathrm{H}$ pylori-infected patients, where they compared the efficacy of LOAD (levofloxacin, 250mg once daily; omeprazole, $40 \mathrm{mg}$ once daily; nitazoxanide $500 \mathrm{mg}$ twice daily with meals; doxycycline, $100 \mathrm{mg}$ once daily) for 7 or 10 days to LAC (lansoprazole 30mg; amoxicillin $1 \mathrm{~g}$ twice daily; clarithromycin $500 \mathrm{mg}$, twice daily for 10 days.

The eradication rate of LOAD 10 and 7 reported in the Basu et al. 2011 study (90\% and 89.4\%) respectively .( Basu PP, et al., 2011)

Also, in the current study anaemia reported in $14.6 \%$ (13/89 patients ) and thrombocytopenia reported in 7.9\% (7/89 patients ) before eradication of HP .

These were correlated to gold, et al. 2014 who confirmed the role of HP in unexplained iron deficiency anaemia (IDA) . (Gold BD,and Elfant AB. 2014)

However, few studies show a clear association between HP infection and idiopathic thrombocytopenia (ITP) with IDA. (Kuku I,et al., 2009)

After eradication of HP there was increase in haemoglobin and platletes in 5.6\%(5/89 patients ) and 3.3\% (3/89 ptients ) respectevly .

These results correlated to, Yamanouchi, et al .2014

Who showed efficacy of HP eradication in treatment of refractory IDA. (Yamanouchi J, et al., 2014)

Also these results agreed with, Morita, et al. 2009 who showed that eradication of HP was an effective treatment for IDA, but not for ITP.( Morita $\mathrm{R}$, et al., 2009)

\section{Summary and Conclusion}

Dyspepsia refers to several symptoms of the upper gastrointestinal tract mainly belonging to three patterns: refluxlike, ulcer-like, and dysmotility-like dyspepsia.

Dyspepsia occurs in approximately $25 \%$ of the population each year. It is responsible for substantial health care costs and considerable time lost from work .

Dyspepsia is characterized by epigastric discomfort or pain with upper abdominal heaviness or fullness, belching or regurgitation, bloating, early satiation, heart burn, food intolerance and nausea or vomiting.

In some patients, no specific cause is identifiable for dyspepsia. This group is labeled as functional or non-ulcer dyspepsia (NUD). The major causes of dyspepsia are peptic ulcer disease, gastro-esophageal reflux disease, malignancy and functional dyspepsia.

Helicobacter pylori (H. pylori) is an organism that has been recognized as a major cause of gastritis and is associated with duodenal ulcer disease, gastric ulcer disease,gastric lymphoma, and gastric cancer in human.

Therefore, it is of the utmost importance to eradicate the pathogen to reduce $\mathrm{H}$. pylori-related complications.

The worldwide increasing resistance of H.pylori towards different antibiotics may affect therapeutic management in different countries.

This search was to study prevalence of Helicobacter pylori in dyspeptic patients and outcome of load regimen on its eradication. After obtaining the approval of the ethical committee at El-Fayoum faculty of medicine and written informed consents of patients, this prospective study was carried out on 100 patients with dyspepsia who underwent upper gastrointestinal endoscopy in El-Fayoum general hospital after evaluating them by history and clinical examination. Urease positive patients $(\mathrm{n}=$ 
89) had been evaluated to LOAD regimen with extended duration (14 days) .

In the current study age ranged from 19-58 years . the Mean \pm SD was $36.6 \pm$ 10.2. females were 58 patients $(58 \%)$ and Males were 42 patients (42\%).

Presenting complaints were epigastric pain, nausea and vomiting 46 (46\%), only epigastric pain $26(26 \%)$ and nausea and/or vomiting were $28(28 \%)$.

Results of 100 dyspeptic patients revealed $16(16 \%)$ anaemic and $8(8 \%)$ thrombocytopenic . elevated liver enzymes in $10(10 \%)$ and elevated FPS 4(4\%) bilirubin elevated in $4(4 \%)$.

$$
\text { As regard } \mathrm{u} / \mathrm{s} \text { : }
$$

Hepatosplenomegally 9(9\%), and just bright liver 15(15\%).

According to HP stool Ag test there were $87(87 \%)$ positive and $13(13 \%)$ negative.

According to multiple gastric biopsies histopathology 90 (90\%) Were positive and $10(10 \%)$ were negative.

According to rapid urease test 89 (89\%) were positive and $11(11 \%)$ were negative for H.pylori infection.

Accuracy of $\mathrm{H}$. pylori antigen when compared to biopsy:

According to our study sensitivity was 93.3 (86.2-96.9) and specificity 70 (39.7-89.2) and PPV 96.6 (90.4-98.8) and NPV53.9 (29.1-76.8) and accuracy was 91 (83.895.2).

Accuracy of $\mathrm{H}$. pylori antigen when compared to urease test:

According to our study sensitivity was 93.2 (86.1-96.9) and Specificity was 63.6 (35.484.8) and PPV95.4 (88.8-98.2) and NPV was $53.9(29.1-76.8)$ and accuracy was 90 (82.6-94.5).

Laboratory investigations of study participants before treatment $(\mathrm{N}=89)$ :

Anaemic13(14.6\%), thrombocytopen ic7(7.9),elevated liver enzymes $6(6.7 \%)$,elevated t.bilirubin $2(2.2 \%)$ and FPS 3(3.4\%).

Laboratory investigations of study participants after treatment $(\mathrm{N}=89)$ :

Anaemic 8 (9\%), thrombocytopenic 4 (4.5\%), elevated Liver enzymes 16
(18\%), elevated bilirubin 9 (10.1) FPS 3 (3.4).

The Rate of eradication of H.pylori after LOAD regimen on H.pylori infected patients was $\mathbf{7 9}(\mathbf{8 8 . 8 \%})$ eradicated and 10(12.2\%) non-eradicated. so we conclude that dyspepsia is a very common upper gastrointestinal disorder and epigastic pain , nausea and vomiting were the most common complaints then nausea, vomiting and epigastric pain separetly and most cases were related to organic causes like NSAIDS and helicobacter pylori infections and the rest of cases were due to functional GIT disorders.

H. pylori prevalence was very high in dyspeptic patients and many H.pylori treatment regimens eradication rate still low or decreasing in efficacy mostly due to antibiotic resistance . because Load regimen was highly effective and safe and the carcinogenicity of H.pylori and its extraintestinal manifestetions. it is highly recommended to screen for H.pylori especially those with dyspepsia, also For H.pyloi infected patients LOAD regimen is highly recommended due to its high eradication rate and safety .

Dyspepsia is a very common upper gastrointestinal disorder and epigastic pain ,nausea and vomiting were the most common complaints then nausea, vomiting and epigastric pain separetly and most cases were related to organic causes like NSAIDS and/or helicobacter pylori infections. The rest of cases were due to functional GIT disorders .

H. pylori prevalence was very high in dyspeptic patients and many H.pylori treatment regimens eradication rate still low or decreasing in efficacy mostly due to antibiotic resistance . and safe

Load regimen was highly effective

\section{References :}

Ali BM, Ameen KH, Hawrami FHR, et al.: Endoscopic findings in dyspeptic patients and their relation to risk factors. Global Journal for Research Analysis, 2015; 4:65-6 
Basu PP, Rayapudi K and Pacana T, et al. A randomized study comparing levofloxacin, omeprazole, nitazoxanide, and doxycycline versus triple therapy for the eradication of Helicobacter pylori. Am J Gastroenterol 2011;106:1970-5.

Carothers JJ, Bruce MG, Hennessy TW, et al. The relationship between previous fluoroquinolone use and levofloxacin resistance in Helicobacter pylori infection. Clin Infect Dis 2007;44:e5-8.

Chey WD and Wong BCY. Practice Parameters Committee of the American College of GastroenterologyManagement of Helicobacter pylori Infection.Am J Gastroenterol 2007;102:1808-25.

De Francesco v, Worldwide $\mathrm{H}$. pyloriantibiotic resistance: a systematic review. J Gastrointestin Liver Dis.2010;194:409-14.

Faintuch JJ, Silva FM, Rodriguez TN, et $a \boldsymbol{a l}$ : Endoscopic findings in uninvestigated dyspepsia Bio Med Central Gastroenterology, 2014; 14:19.

Flier, SN and S, Rose (2006). "Is functional dyspepsia of particular concern in women? A review of gender differences in epidemiology, pathophysiologic mechanism, clinical presentation and management". Am J Gastroenterol. 101: S644-S653.

Gado A, Ebeid B , Abdelmohsen A, et al.: Endoscopic evaluation of patients with dyspepsia in a secondary referral hospital in Egypt. Alexandria Journal of Medicine, 2015; 51, 179-184.

García-Alonso FJ, Tejero MH, Benitob ER, et al.: Implementation and evaluation of early gastroscopy for patients with dyspepsia and warning signs in primary care. Gastroenterología y Hepatología, 2016; 1081:1-8.

GisbertJP,: $\quad$ Proton pump inhibitor,clarithromycin and either amoxycillin or nitroimidazole: a metaanalysisof eradication of Helicobacter pylori. Aliment PharmacolTher. 2000;14:1319-28.

Gisbert JP, de la Morena F , Abraira V. Accuracy of monoclonal stool antigen test for the diagnosis of $\mathrm{H}$. pylori infection: a systematic review and meta-analysis. Am J Gastroenterl 2006; 101: 1921-1930

Gold BD, Gilger MA and Czinn SJ. New Diagnostic Strategies for Detection of Helicobacter pylori Infection in Pediatric Patients. Gastroenterol Hepatol (N Y). 2014 Dec;10(12 Suppl 7):1-19

Hojo M,. Pooled analysis on the efficacy othe second-line treatment regimens for Helicobacter pylori infection.Scand $\mathbf{J}$ Gastroenterol 2001;36:690-700.

Hunt RH, Xiao SD, Megraud F, et al.; World Gastroenterology Organization : Helicobacter Pylori in Developing Countries. World Gastroenterology Organisation Global Guideline. $J$ Gastrointestin Liver Dis. 2011; 20(3):299304

James K. Y. Hooi, Wan Ying Lai, Wee Khoon Ng, et al. : AGA jornal ,Gastroenterology 2017;153:420-429

J. Borg, O. Melander, L. Johansson, et al.: "Gastroparesis is associated with oxytocin deficiency, oesophageal dysmotility with hyperCCKemia, and autonomic neuropathy with hypergastrinemia,"BMCGastroenterology, vol. 9, article 17, 2009.

Khedmat H, Karbasi-Afshar R, Hossein Khedmat, et al. (2013). Helicobacter pylori infection in the general population: A middle eastern perspective. Caspian $J$ Intern Med, 4, 745-53.

Kuku I, Kaya E, Yologlu S, et al. (2009): Platelets counts in adults with iron deficiency anemia. Platelets 20:401-405

LiangCM,.Levofloxacin-containing secondlinanti-Helicobacter pylori eradication in Taiwanese realworldpractice. Biomed J 2014;375:326-0.

Liou JM, Levofloxacin sequential therapy vslevofloxacin triple therapy in the secondline treatment of Helicobacterpylori: a randomized trial. Am J Gastroenterol 2016;1113:381-7.

Malfertheiner P,. Current concepts in themanagement of Helicobacter pylori infection - the Maastricht 2-2000 Consensus Report. Aliment PharmacolTher 2002;16:16780. 
Megraud F. Helicobacter pylori resistanceto antibiotics in Europe and its relationship to antibiotic consumption.Gut 2013;62:34-2.

Molina-Infa nte J, Lucendo AJ , Angueira T, et al. Optimised empiric tripleand concomitant therapy for Helicobacter pylori eradication in clinical practice: the OPTRICON study. Aliment Pharmacol Ther 2015;41:581-9.

Morita R, Hashino R, Okada K, et al. (2009) : Iron deficiency anemia successfully treated by Helicobacter pylori eradication in a patient with idiopathic thrombocytopenic purpura. Rinsho Ketsueki 50:1655-1657,

Napthali K, Koloski N, Walker MM, et al.: Women and functional dyspepsia ,Womens Health, 2016.

Paul S, Paljor HP, Kumar V, et al.: Clinical and endoscopic evaluation of patients with dyspepsia. Indian Journal of Medical Specialties, 2015; 6:98-101.

Peek MRJr and Blaser MJ. Helicobacter pylori and gastrointestinal tract adenocarcinomas. Nat Rev Cancer 2002;2:28-37

Romano M, Lovene MR, Russo MI, et al. Failure of first-line eradication treatment significantly increases prevalence of antimicrobial-resistant Helicobacter pylori clinical isolates. J Clin Pathol 2008;61:1112-5.

Shrestha R, Karki S ,Pandey B, et al.: Upper gastrointestinal endoscopy findings in patient presenting with dyspepsia. Journal of Patan Academy of Health Sciences, 2015; 2(2):19-22.

Tacikowski T, Bawa S, Gajewska D, et al.: Current prevalence of Helicobacter pylori infection in patients with dyspepsia treated in Warsaw, Poland. Gastroenterology, 2017; 12(2):135-139.

Tiwari SK, Khan AA, Nallari P : Helicobacter pylori: "A benign fellow traveler or unwanted inhabitant". J Med Allied Sci 2011;1(1):2-6

Vaira D, Vakil N, Gatta L, et al. Accuracy of a new ultrafast rapid urease test to diagnosis Helicobacter pylori infection in 1000 consecutive dyspeptic patients. Aliment Pharmacol Ther 2010; 31: 331-338

Vasiliou C, Xiromeritou V, Kafiri G, et al.: Endoscopic and histological findings and helicobacter pylori status in patients with reflux and/or dyspeptic symptoms. Gastroenterology Nurses and Associates, 2014; 37:431-438.

Yamanouchi J, Azuma T, Yakushijin Y, et al.: (2014) Dramatic prompt efficacy of Helicobacter pylori eradication in the treatment of severe refractory iron deficiency anemia in adult. Ann Hematol 93:1779-1780

Yao-Kuang Wang, Fu-Chen Kuo, Chung-Jung Liu, et al. : World $J$ Gastroenterol 2015 October 28; 21(40): 11221-1123

Yilmaz O, Demiray E. Clinical role and importance of fluorescence in situ hybridization method in diagnosis of $\mathrm{H}$ pylori infection and determination of clarithromycin resistance in $\mathrm{H}$ pylori eradication therapy. World J Gastroenterol 2007; 13: 671-675.

Zullo A,. Second-line and rescuetherapies for Helicobacter pylori eradication in clinical practice. JGastrointestin Liver Dis 2010;192:131-4. 